\title{
Revised Newtonian Formula of Gravity and Equation of Cosmology in Flat Space-Time Transformed from Schwarzschild Solution
}

\author{
Xiaochun Mei, Ping Yu \\ Institute of Innovative Physics in Fuzhou, Department of Physics, Fuzhou University, Fuzhou, China \\ Email: ycwlyjs@yeah.net, yupingpingyu@yahoo.com
}

Received January 3, 2012; revised February 7, 2012; accepted February 16, 2012

\begin{abstract}
By transforming the geodesic equation of the Schwarzschild solution of the Einstein's equation of gravity field to flat space-time for description, the revised Newtonian formula of gravity is obtained. The formula can also describe the motion of object with mass in gravity field such as the perihelion precession of the Mercury. The space-time singularity in the Einstein's theory of gravity becomes the original point $r=0$ in the Newtonian formula of gravity. The singularity problem of gravity in curved space-time is eliminated thoroughly. When the formula is used to describe the expansive universe, the revised Friedmann equation of cosmology is obtained. Based on it, the high red-shift of Ia supernova can be explained well. We do not need the hypotheses of the universe accelerating expansion and dark energy again. It is also unnecessary for us to assume that non-baryon dark material is 5 - 6 times more than normal baryon material in the universe if they really exist. The problem of the universal age can also be solved well. The theory of gravity returns to the traditional form of dynamic description and becomes normal one. The revised equation can be taken as the foundation of more rational cosmology.
\end{abstract}

Keywords: General Relativity; Schwarzschild Metric; Cosmology; Friedmann Equation; Newtonian Gravity; Supernova; Hubble Constant; Dark Material; Dark Energy; Universe Accelerating Expansion; Universe Age

\section{Introduction}

Established on the foundation of curved space-time, Einstein's theory of gravity is the dominate theory at present. However, Einstein's theory has some difficulties hard to be overcome such as the problems of normalization, singularity and uniqueness of gravity field's energy and so on. In addition, it is difficulty to solve the non-linear Einstein's equation of gravity field. It is always attractive to reestablish the theory of gravity in flat space-time without these troubles. Since the 1940 's, many people has tried and many theories had been proposed [1,2]. These theories are consistent with Einstein's one under the condition of weak fields, but are different in strong fields. Meanwhile, these theories also have some problems hard to be overcome.

The standard theory of cosmology faces many principle difficulties at present. As is proved below, the problems originate from the Friedmann equation which is unsuitable to describe the high speed expansion of the universe. The reason is that two simplified and improper conditions were used in the deduction of the Friedmann equation. They are the R-W metric and static energy momentum tensor. At present, the R-W metric is considered with constant spatial curvature. However, the author had proved that strictly based on the curvature formula of the Riemannian geometry, when the scalar factor $R(t)$ changes with time, the R-W metric has no constant curvature [3]. The common understanding about the spatial curvature of the R-W metric is wrong. This idea would impose great influence on cosmology. Due to this result, many conclusions in the cosmology such as the densities of dark material and dark energy should be re-estimated.

It is proved further in this paper that the R-W metric leads to the Galileo's transformation of light's velocity, instead of the Einstein's transformation. So the R-W metric is not relativity metric and unsuitable to be taken as the basic space-time framework of modern cosmology.

Meanwhile, because relative velocities exist between materials and observers in the expansive universe, the equation of cosmology should use dynamic energy momentum tensor, rather than static one as commonly used in the current cosmology.

In fact, E. A. Milne pointed out in 1943 that the Friedmann equation of cosmology could be deduced based on the Newtonian formula of gravity [4]. It means that the 
Friedmann equation is equivalent to the Newtonian theory actually. It is only suitable for describing the process of low speed expansion of the universe, but not for the process of high speed expansion.

However, it is proved in this paper that if dynamic energy momentum tensor is used, the equation of cosmology would become very complex, so that it can not be solved actually. The pioneer of cosmology must have considered this problem and had to use static energy momentum tensor. In the early stage of cosmology, the Friedmann equation seemed to be appreciable because the expansive speed observed was low. When cosmology develops to present level, we observe the high speed expansion. In this case, the Friedmann equation becomes unsuitable for the problems such as the high red-shift of supernova. We have to find more precise method to describe them.

It is proved in this paper that by transforming the geodesic equation of the Schwarzschild solution of the Einstein's equation of gravity field to flat space-time, the revised Newtonian formula of gravity can be obtained. The formula can well describe the perihelion precession of the Mercury. The space-time singularities in the Einstein's theory of gravity become the point $r=0$ in the revised Newtonian formula of gravity. We have no the trouble of singularities again.

When the revised formula is used to describe the expansive universe, we obtain the revised Friedmann equation. Based on it, the high red-shift of supernova can be explained well without the hypotheses of the universal accelerating expansion and dark energy. Many problems including the universe age to be too small can also be resolved well. In this way, we can get rid of the current puzzle situation of cosmology completely.

\section{Revised Newtonian Formula of Gravity Based on the Schwarzschild}

\subsection{Revised Newtonian Formula of Gravity}

According to general relativity, the Schwarzschild metric (external solution) is

$$
\mathrm{d} s^{2}=c^{2}\left(1-\frac{\alpha}{r}\right) \mathrm{d} t^{2}-\left(1-\frac{\alpha}{r}\right)^{-1} \mathrm{~d} r^{2}-r^{2}\left(\sin ^{2} \theta \mathrm{d} \varphi^{2}+\mathrm{d} \theta^{2}\right)
$$

Here $\alpha=2 G M_{0} / c^{2}$. Let $\theta=\pi / 2$ and substitute (1) into the equation of geodetic line, we have the integrals

$$
c\left(1-\frac{\alpha}{r}\right) \frac{\mathrm{d} t}{\mathrm{~d} s}=\varepsilon \quad r^{2} \frac{\mathrm{d} \varphi}{\mathrm{d} s}=\frac{L}{c}
$$

Here $\varepsilon$ and $L$ are constants. By cancelling ds from the formulas, we can obtain

$$
r^{2}\left(1-\frac{\alpha}{r}\right)^{-1} \frac{\mathrm{d} \varphi}{\mathrm{d} t}=\frac{L}{\varepsilon}
$$

We define

$$
\mathrm{d} \tau=\left(1-\frac{\alpha}{r}\right) \mathrm{d} t
$$

In which $\tau$ is eigen time, $t$ is coordinate time. Then, let $\varepsilon=1$, we have from (2)

$$
c \mathrm{~d} \tau=\mathrm{d} s
$$

Then, (3) becomes

$$
r^{2} \frac{\mathrm{d} \varphi}{\mathrm{d} \tau}=L
$$

Here $L$ is the angular momentum of unit mass. (6) is just the conservation formula of angel momentum.

We only discuss the motion of particles with mass in gravitational field. By considering (6), we write (1) as

$$
\left(1-\frac{\alpha}{r}\right)\left(\frac{\mathrm{d} t}{\mathrm{~d} \tau}\right)^{2}-\left(1-\frac{\alpha}{r}\right)^{-1}\left(\frac{\mathrm{d} r}{c \mathrm{~d} \tau}\right)^{2}-r^{2}\left(\frac{\mathrm{d} \varphi}{c \mathrm{~d} \tau}\right)^{2}=1
$$

By considering (4) and (6), the formula above can be written as

$$
\left(\frac{\mathrm{d} r}{\mathrm{~d} \tau}\right)^{2}=\frac{c^{2} \alpha}{r}\left(1-\frac{L^{2}}{\alpha c^{2} r}+\frac{L^{2}}{c^{2} r^{2}}\right)
$$

Taking the differential of (8) about $\mathrm{d} \tau$, we get

$$
\frac{\mathrm{d}^{2} r}{\mathrm{~d} \tau^{2}}-\frac{L^{2}}{r^{3}}=-\frac{c^{2} \alpha}{2 r^{2}}\left(1+\frac{3 L^{2}}{c^{2} r^{2}}\right)
$$

Note that all quantities in (9) are defined in curved space-time. According to the theory of the non-Euclidean geometry, although we can not transform whole metric of curved space-time into that of flat space-time in general, we can always transform the geodetic line described in curved space-time into that in flat space. Let $r_{0}, \phi_{0}$ and $t_{0}$ represent the space-time coordinates of flat spacetime, due to the invariability of $\mathrm{ds}^{2}$, we have

$$
\begin{aligned}
& \mathrm{d} s^{2}=c^{2} \mathrm{~d} t_{0}^{2}-\mathrm{d} r_{0}^{2}-r_{0}^{2} \mathrm{~d} \varphi_{0}^{2} \\
= & c^{2}\left(1-\frac{\alpha}{r}\right) \mathrm{d} t^{2}-\left(1-\frac{\alpha}{r}\right)^{-1} \mathrm{~d} r^{2}-r^{2} \mathrm{~d} \varphi^{2}
\end{aligned}
$$

We see that the forms of third items on the two sides of the second equal sign of the formula above are completely the same. So we can take $r_{0}=r, \varphi_{0}=\varphi$ and get the relation between times $t_{0}$ and $t$

$$
c^{2} \mathrm{~d} t_{0}^{2}=c^{2}\left(1-\frac{\alpha}{r}\right) \mathrm{d} t^{2}+\left[1-\left(1-\frac{\alpha}{r}\right)^{-1}\right] \mathrm{d} r^{2}
$$

by considering (4), we get and from (8)

$$
\mathrm{d} r=c\left(1-\frac{\alpha}{r}\right) \sqrt{\frac{\alpha}{r}\left(1-\frac{L^{2}}{\alpha c^{2} r^{3}}+\frac{L^{2}}{c^{2} r^{2}}\right)} \mathrm{d} t
$$

Substituting it into (11), we get 


$$
\mathrm{d} t_{0}=\sqrt{\left(1-\frac{\alpha}{r}\right)\left(1-\frac{\alpha^{2}}{r^{2}}+\frac{\alpha L^{2}}{c^{2} r^{3}}-\frac{\alpha^{2} L^{2}}{c^{2} r^{4}}\right)} \mathrm{d} t
$$

Comparing with (4), we have

$$
\mathrm{d} \tau=\left(1-\frac{\alpha}{r}\right)^{\frac{1}{2}}\left(1-\frac{\alpha^{2}}{r^{2}}+\frac{\alpha L^{2}}{c^{2} r^{3}}-\frac{\alpha^{2} L^{2}}{c^{2} r^{4}}\right)^{-\frac{1}{2}} \mathrm{~d} t_{0}
$$

Because we have taken $r_{0}=r$, all quantities on the right side of (14) have been defined in flat space-time. Note that in the classical Newtonian theory of gravity, at the directions of $\boldsymbol{e}_{r}$ and $\boldsymbol{e}_{\phi}$ in plane polar coordinates system, the partial motion equations of unit mass are individually

$$
\begin{aligned}
& \frac{\mathrm{d}^{2} r}{\mathrm{~d} t^{2}}-r\left(\frac{\mathrm{d} \varphi}{\mathrm{d} t}\right)^{2}=-\frac{c^{2} \alpha}{2 r^{2}} \\
& \frac{1}{r} \frac{\mathrm{d}}{\mathrm{d} r}\left(r^{2} \frac{\mathrm{d} \varphi}{\mathrm{d} t}\right)=0 \quad \text { or } \quad r^{2} \frac{\mathrm{d} \varphi}{\mathrm{d} t}=L
\end{aligned}
$$

Substitute (16) into (15), we get

$$
\frac{\mathrm{d}^{2} r}{\mathrm{~d} t^{2}}-\frac{L^{2}}{r^{3}}=-\frac{c^{2} \alpha}{2 r^{2}}
$$

Comparing with (9) and let $t \rightarrow \tau$, except the revised item in the bracket of (9), we see that the forms of (9) and (17) are completely similar. So we can write (9) as the following vector equation

$$
m_{0} \frac{\mathrm{d}^{2} \boldsymbol{r}}{\mathrm{d} \tau^{2}}=-G M m_{0}\left(1+\frac{3 L^{2}}{c^{2} r^{2}}\right) \frac{\boldsymbol{r}}{r^{3}}
$$

Let $u=1 / r$ and consider (6), the formula above can be transformed to

$$
\frac{\mathrm{d}^{2} u}{\mathrm{~d} \varphi^{2}}+u=\frac{G M}{L^{2}}\left(1+\frac{3 L^{2} u^{2}}{c^{2}}\right)
$$

This formula is the one used to describe the perihelion precession of the Mercury in general relativity. In the deduction process above, we use the equation of geodetic line (2). It means that we transform the equation of geodetic line into the revised formula of the Newtonian gravity, in stead of transforming whole curved space-time to flat space-time. But it is enough for us to describe an object's motion in gravity field.

Now let's prove that the effect of special relativity has been taken into account in (18). From (8), (12) and (14), we can obtain

$$
\begin{aligned}
V_{r}^{2}= & \left(\frac{\mathrm{d} r}{\mathrm{~d} t_{0}}\right)^{2}=\left(\frac{\mathrm{d} r}{\mathrm{~d} \tau} \frac{\mathrm{d} \tau}{\mathrm{d} t_{0}}\right)^{2}=\frac{c^{2} \alpha}{r}\left(1-\frac{\alpha}{r}\right) \\
& \times\left(1-\frac{L^{2}}{\alpha c^{2} r}+\frac{L^{2}}{c^{2} r^{2}}\right)\left(1-\frac{\alpha^{2}}{r^{2}}+\frac{\alpha L^{2}}{c^{2} r^{3}}-\frac{\alpha^{2} L^{2}}{c^{2} r^{4}}\right)^{-1}
\end{aligned}
$$

$$
\begin{aligned}
V_{\varphi}^{2}= & \left(r \frac{\mathrm{d} \varphi}{\mathrm{d} t_{0}}\right)^{2}=\left(r \frac{\mathrm{d} \varphi}{\mathrm{d} \tau} \frac{\mathrm{d} \tau}{\mathrm{d} t_{0}}\right)^{2} \\
= & \frac{L^{2}}{r^{2}}\left(1-\frac{\alpha}{r}\right)\left(1-\frac{\alpha^{2}}{r^{2}}+\frac{\alpha L^{2}}{c^{2} r^{3}}-\frac{\alpha^{2} L^{2}}{c^{2} r^{4}}\right)^{-1} \\
V^{2}= & V_{r}^{2}+V_{\varphi}^{2}=\frac{c^{2} \alpha}{r}\left(1-\frac{\alpha}{r}\right) \\
& \times\left(1+\frac{L^{2}}{c^{2} r^{2}}\right)\left(1-\frac{\alpha^{2}}{r^{2}}+\frac{\alpha L^{2}}{c^{2} r^{3}}-\frac{\alpha^{2} L^{2}}{c^{2} r^{4}}\right)^{-1} \\
1-\frac{V^{2}}{c^{2}} & =\left(1-\frac{\alpha}{r}\right)\left(1-\frac{\alpha^{2}}{r^{2}}+\frac{\alpha L^{2}}{c^{2} r^{3}}-\frac{\alpha^{2} L^{2}}{c^{2} r^{4}}\right)^{-1}
\end{aligned}
$$

Comparing with (14), we get

$$
\mathrm{d} \tau=\sqrt{1-\frac{V^{2}}{c^{2}}} \mathrm{~d} t_{0}
$$

This is just the formula of time delay in special relativity. The result verifies the rationality of (18). Let $t_{0} \rightarrow t$ at last, we write (18) as

$$
\frac{\mathrm{d} \boldsymbol{p}}{\mathrm{d} t}=-G M m_{0} \sqrt{1-\frac{V^{2}}{c^{2}}}\left(1+\frac{3 L^{2}}{c^{2} r^{2}}\right) \frac{\boldsymbol{r}}{r^{3}}=\boldsymbol{F}
$$

It is the revised Newtonian formula of gravity based on general relativity. In the formula, $m_{0}$ is the static mass of moving particle and the center static mass $M_{0}$ has spherical symmetry. Angle momentum makes gravity larger but speed makes it smaller. The result is equivalent to replace particle's static mass with following effective mass in the Newtonian theory

$$
m=m_{0} \sqrt{1-\frac{V^{2}}{c^{2}}}\left(1+\frac{3 L^{2}}{c^{2} r^{2}}\right)
$$

We can call $m$ as the motion mass of gravity which is related to object's speed and angle momentum.

\subsection{The Motion of Particle in Gravitational Field with Spherical Symmetry}

For simplicity, we only discuss the motion of a particle moves along the radius vector direction with $L=0$. In this case, by considering (23), (25) becomes

$$
\boldsymbol{F}=\frac{\mathrm{d} \boldsymbol{p}}{\mathrm{d} t}=-\frac{G M m_{0}}{\sqrt{1+\alpha / r}} \frac{\boldsymbol{r}}{r^{3}}
$$

by multiplying $\mathrm{d} \boldsymbol{r}$ on both sides of (27), the potential energy of the particle in gravitational field is

$$
\begin{aligned}
U(r) & =-\int \boldsymbol{F} \cdot \mathrm{d} \boldsymbol{r}=\int \frac{m_{0} c^{2} \alpha}{2 \sqrt{1+\alpha / r}} \frac{\boldsymbol{r}}{r^{3}} \cdot \mathrm{d} \boldsymbol{F} \\
& =-m_{0} c^{2} \sqrt{1+\frac{\alpha}{r}}+A_{1}
\end{aligned}
$$


The dynamic energy of particle is

$$
\begin{aligned}
T= & \int \frac{\mathrm{d} \boldsymbol{p}}{\mathrm{d} t} \cdot \mathrm{d} \boldsymbol{r}=\int \frac{\mathrm{d} \boldsymbol{p}}{\mathrm{d} t} \cdot \frac{\mathrm{d} \boldsymbol{r}}{\mathrm{d} t} \mathrm{~d} t=\int \boldsymbol{V} \cdot \mathrm{d} \boldsymbol{p}=\boldsymbol{V} \cdot \boldsymbol{p} \\
& -\int \boldsymbol{p} \cdot \mathrm{d} \boldsymbol{V}=\frac{m_{0} V^{2}}{\sqrt{1-V^{2} / c^{2}}}+m_{0} c^{2} \sqrt{1-\frac{V^{2}}{c^{2}}}+A_{2}
\end{aligned}
$$

when $r \rightarrow \infty$, we have $V=0$ and $A_{2}=-m_{0} c^{2}$. So the law of energy conservation of a particle in the gravitational field can be written as

$$
\begin{aligned}
T+U= & \frac{m_{0} V^{2}}{\sqrt{1-V^{2} / c^{2}}}+m_{0} c^{2}\left(\sqrt{1-\frac{V^{2}}{c^{2}}}-1\right) \\
& -m_{0} c^{2}\left(\sqrt{1+\frac{\alpha}{r}}-1\right)=0
\end{aligned}
$$

when $\alpha / r \ll 1$ and $V \ll c$, we get the classic law of energy conservation in the Newtonian theory of gravity

$$
\frac{m_{0} V^{2}}{2}-\frac{G M m_{0}}{r}=0
$$

In the situation of $L \neq 0$, we calculate the problem in the weak field with $\alpha / r \ll 1$. By keeping items with the orders up to $r^{-2}$, we have

$$
\begin{aligned}
U(r)= & -\int \boldsymbol{F} \cdot \mathrm{d} \boldsymbol{r}=\int \frac{m_{0} c^{2} \alpha}{2 \sqrt{1+\alpha / r}}\left(1+\frac{3 L^{2}}{c^{2} r^{2}}\right) \frac{\boldsymbol{r}}{r^{3}} \cdot \mathrm{d} \boldsymbol{r} \\
= & -m_{0} c^{2}\left\{\sqrt{1+\frac{\alpha}{r}}-\frac{3 L^{2}}{c^{2} \alpha^{2}}\left(1+\frac{\alpha}{r}\right)\right. \\
& \left.\times\left[\frac{1}{6}\left(1+\frac{\alpha}{r}\right)^{2}-\frac{2}{3}\left(1+\frac{\alpha}{r}\right)^{1 / 2}+1\right]\right\}+A_{1}
\end{aligned}
$$

So the law of energy conservation is

$$
\begin{aligned}
E= & \frac{m_{0} V^{2}}{\sqrt{1-V^{2} / c^{2}}}+m_{0} c^{2}\left\{\sqrt{1-\frac{V^{2}}{c^{2}}}-\sqrt{1+\frac{\alpha}{r}}\right. \\
& \left.+\frac{3 L^{2}}{c^{2} \alpha^{2}}\left(1+\frac{\alpha}{r}\right)\left[\frac{1}{6}\left(1+\frac{\alpha}{r}\right)^{2}-\frac{2}{3}\left(1+\frac{\alpha}{r}\right)^{1 / 2}+1\right]\right\}
\end{aligned}
$$

Here $E$ is a constant.

Now let's discuss the motion of a particle in the gravity field. Suppose that a particle falls freely along the radium direction of gravity field, its velocity and acceleration are individually

$$
\begin{aligned}
& V=\frac{\mathrm{d} r}{\mathrm{~d} t}=-c \sqrt{\frac{\alpha}{r}}\left(1+\frac{\alpha}{r}\right)^{-1 / 2} \\
& a=-\frac{1}{2} \frac{c^{2} \alpha}{r^{2}}\left(1+\frac{\alpha}{r}\right)^{-2}
\end{aligned}
$$

when $r \rightarrow \infty$, we have $V=0$ and $a=0$. Suppose that the particle is at point $r=r_{0}$ when $t=0$, by the integral of (34), we get

$$
c t=\frac{2}{3 \sqrt{\alpha}}\left[\left(r_{0}+\alpha\right)^{3 / 2}-(r+\alpha)^{3 / 2}\right]
$$

It is obvious that every thing is normal within the region $r>0$. The particle is monotonously accelerated by gravitation. There is no any singularity in the whole spacetime. When particle is at the original point $r=0$, we have

$$
\begin{aligned}
& V=-\lim _{x \rightarrow \infty} \frac{c \sqrt{\alpha / r}}{\sqrt{1+\alpha / r}} \rightarrow-c \\
& a=-\lim _{x \rightarrow \infty} \frac{c^{2} x^{2}}{2 \alpha(x+1)^{2}} \rightarrow-\frac{c^{2}}{2 \alpha} \\
& F=-\lim _{x \rightarrow \infty} \frac{c^{2} x^{2}}{2 \alpha(x+1)} \rightarrow-\lim _{x \rightarrow \infty} \frac{c^{2} x}{2 \alpha} \rightarrow-\infty
\end{aligned}
$$

It indicates that the speed of particle tends to have light's speed in vacuum at point $r=0$. Acceleration is also finite. So within the region $0<r \leq \infty$, the motion of particle with static mass is continuous. Only at point $r=0$, the force acted on particles becomes infinite. But this kind of singularity appears in any theories in which particles are considered with infinite small size, and have nothing to do with space-time singularity. The singularity of the Schwarzschild solution is eliminated.

\section{The Fiedmann Equation of Cosmology Needs Relativity Revision}

\subsection{The Fiedmann Equation is Equivalent to the Newtonian Theory of Gravity}

The Fiedmann equation of cosmology is based on the Einstein's equation of gravity. Because the equation is too complex to solve, two simplified conditions are used. One is the R-W metric and another is the static energy momentum tensor. Using them, we obtain from the Einstein's equation of gravity

$$
\begin{aligned}
& \frac{\ddot{R}}{R}=-\frac{4 \pi G}{3}(\rho+3 p) \\
& \frac{\ddot{R}}{R}+\frac{2 \dot{R}^{2}}{R^{2}}+\frac{2 \kappa}{R^{2}}=4 \pi G(\rho-p)
\end{aligned}
$$

Here $R(t)$ is scalar factor, $\kappa$ is curvature constant factor, $\rho$ is the universe material density and $p$ is the intensity of pressure. By eliminating $\ddot{R}$ form (39), we obtain the Fiedmann equation

$$
\frac{\dot{R}^{2}}{R^{2}}+\frac{\kappa}{R^{2}}=\frac{8 \pi G}{3} \rho
$$

Cosmic constant has not been considered in (39) and (40). We often either take it as zero, or combine it with effective material density for convenience. 
However, British physicist E. A. Milne proved in 1943 that the Fiedmann equation could be deduced simply based on the Newtonian theory of gravity. Though the Fiedmann equation is described in curved space-time and the Newtonian theory of gravity is described in flat space-time, the results are the same actually when we use them to calculate practical problems, especially when we take curvature constant $\kappa=0$. However, the Newtonian theory of gravity is only suitable for the motions with low speeds. For the high speed expansion of the universe, it is unsuitable. The Fiedmann equation needs relativity revision due to this fact.

We now repeat Milne's deduction below. According to the principle of cosmology, the universe can be considered as a huge sphere with uniform and isotropic material distribution. According to the Newtonian theory, gravity acted on a body located at point $r$ inside the sphere is only related to the mass contained in the sphere with radius $r$, having nothing to do with the mass outside the sphere. Suppose that the mass of uniform sphere to be $M=4 \pi r^{3}(t) \rho(t) / 3$, in the direction of sphere radius, the Newtonian equation of gravity is

$$
m \frac{\mathrm{d}^{2} r}{\mathrm{~d} t^{2}}=-\frac{G M m}{r^{2}}=-\frac{4 \pi G m \rho}{3} r
$$

For the expansive sphere, by considering co-moving coordinate $r=R(t) \bar{r}$ in which $\bar{r}$ has nothing to do with time, (41) becomes

$$
\ddot{R}(t)=-\frac{4 G \pi \rho(t)}{3} R(t)
$$

(42) is the same as the first formula of (39) when $p=0$. Because mass is invariable in the expansive process, we have $\rho(t) R^{3}(t)=\rho\left(t_{0}\right) R^{3}\left(t_{0}\right)$, here $t_{0}$ is the time at present. We have

$$
\ddot{R}(t)=\frac{\mathrm{d} \dot{R}(t)}{\mathrm{d} t}=\frac{\mathrm{d} \dot{R}(t)}{\mathrm{d} R(t)} \frac{\mathrm{d} R(t)}{\mathrm{d} t}=\dot{R}(t) \frac{\mathrm{d} \dot{R}(t)}{\mathrm{d} R(t)}
$$

Substituting (43) in (42) and taking the integral, we obtain (40). In this case, integral constant $\kappa$ is equivalent with curvature constant in the $\mathrm{R}-\mathrm{W}$ metric.

It is obvious that (40) is the direct result of the Newtonian theory of gravity, for it dose not contain any revised item of relativity. This is why the standard theory of cosmology is effective for same problems, but is ineffective for other problems such as the high red shift of supernova. The reason is that two simplified conditions are used, so that the Freidmann equation becomes non-relativity theory actually. We discuss these problems below.

\subsection{The R-W Metric Violates the Principle of Invariance of Light's Velocity}

According to the principle of cosmology, the universe is uniform and isotropy. The R-W metric is considered with the biggest space-time symmetry. Its form is

$$
\mathrm{d} s^{2}=c^{2} \mathrm{~d} t^{2}-R^{2}(t)\left(\frac{\mathrm{d} \bar{r}^{2}}{1-\kappa \bar{r}^{2}}+\bar{r}^{2} \mathrm{~d} \theta^{2}+\bar{r}^{2} \sin ^{2} \theta \mathrm{d} \varphi^{2}\right)
$$

In which $\kappa$ is curvature factor. When $\kappa=0$, the metric becomes flat with

$$
\mathrm{d} s^{2}=c^{2} \mathrm{~d} t^{2}-R^{2}(t)\left(\mathrm{d} \bar{r}^{2}+\bar{r}^{2} \mathrm{~d} \theta^{2}+\bar{r}^{2} \sin ^{2} \theta \mathrm{d} \varphi^{2}\right)
$$

For light's motion, we have $\mathrm{d} s=0$. When light moves along radius direction, we have $\mathrm{d} \theta=\mathrm{d} \varphi=0$. According to (45), we obtain

$$
\frac{\mathrm{d} \bar{r}}{\mathrm{~d} t}= \pm \frac{C}{R(t)}
$$

For the light's source fixed at point $\bar{r}$, coordinate $\bar{r}$ does not change with time. But for the light emitted by light's source, coordinate $\bar{r}$ changes with time as described in (46). The velocity of space expansion is

$$
V(t)=\frac{\mathrm{d} r(t)}{\mathrm{d} t}=\dot{R}(t) \bar{r}
$$

By considering (46) and (47), the velocity of light relative to observer located at the original point of reference frame is

$$
\begin{aligned}
V_{c}(t) & =\frac{\mathrm{d} r(t)}{\mathrm{d} t}=\bar{r} \frac{\mathrm{d}}{\mathrm{d} t} R(t)+R(t) \frac{\mathrm{d} \bar{r}}{\mathrm{~d} t} \\
& =\dot{R}(t) \bar{r} \pm c=V(t) \pm c
\end{aligned}
$$

The formula indicates that light's velocity is related to the expansion speed of space and violates the principle of invariance of light's speed.

In fact, at the moment when light is just emitted out, (48) is the Galileo's addition rule of light's velocity. When light moves towards observer, minus sign is taken in (48) so light's speed is less than its speed in vacuum. When the light moves apart from observer, plus sign is taken. In this case, light's speed exceeds its speed in vacuum. Especially, because $\bar{r}$ increases with time, enough long time later, light's speed may greatly exceed its speed in vacuum.

This is not allowed in physics. As we know that the watershed between classical physics and modern physics is just on the invariance principle of light's speed. Because the R-W metric violates this principle, it can not be used as the space-time frame for modern cosmology which is considered as the theory of relativity. Especially when the expansion speed of the universe is great, huge error will be caused.

As for the curve space with $\kappa \neq 0$, let $\mathrm{d} s=0$ and $\mathrm{d} \theta=\mathrm{d} \varphi=0$ in (44), we obtain

$$
\frac{\mathrm{d} \bar{r}}{\mathrm{~d} t}= \pm \frac{c \sqrt{1-\kappa \bar{r}^{2}}}{R(t)}
$$


or

$$
\frac{\mathrm{d} \bar{r}}{\sqrt{1-\kappa \bar{r}^{2}}}= \pm \frac{c \mathrm{~d} t}{R(t)}
$$

On the other hand, as we known that coordinate $\bar{r}$ has no meaning of measurement in curved space. What is meaningful is proper distance. Suppose that an observer stays at the original point of coordinate system, the definition of proper distance for the R-W metric between observer and light's source is [5].

$$
r(t)=R(t) \int_{0}^{\bar{r}} \frac{\mathrm{d} \bar{r}_{1}}{\sqrt{1-\kappa \bar{r}_{1}^{2}}}=R(t) l(\bar{r})
$$

Here $l(\bar{r})$ equivalent to $\bar{r}$ in the flat space. For illuminant material moving in the expansive universe, $l(\bar{r})$ does not change with time. The velocity of illuminant material relative to observer is $V(t)=\dot{R}(t) l(\bar{r})$. By considering (49), the velocity of light emitted by illuminant material moves in the curved space is

$$
\begin{aligned}
V_{c}(t) & =\frac{\mathrm{d} r(t)}{\mathrm{d} t}=\dot{R}(t) \int_{0}^{\bar{r}} \frac{\mathrm{d} \bar{r}_{1}}{\sqrt{1-\kappa \bar{r}_{1}^{2}}}+R(t)\left(\frac{\mathrm{d}}{\mathrm{d} \bar{r}} \int_{0}^{\bar{r}} \frac{\mathrm{d} \bar{r}_{1}}{\sqrt{1-\kappa \bar{r}_{1}^{2}}}\right) \frac{\mathrm{d} \bar{r}}{\mathrm{~d} t} \\
& =\dot{R}(t) l(\bar{r})+\frac{R(t)}{\sqrt{1-\kappa \bar{r}^{2}}} \frac{\mathrm{d} \bar{r}}{\mathrm{~d} t}=V(t) \pm c
\end{aligned}
$$

So (51) still violates the principle of invariance of light's speed. In fact, the four dimensional metric of flat space-time is

$$
\mathrm{ds} s^{2}=c^{2} \mathrm{~d} t^{2}-\left(\mathrm{d} r^{2}+r^{2} \mathrm{~d} \theta^{2}+r^{2} \sin ^{2} \theta \mathrm{d} \varphi^{2}\right)
$$

by using co-moving coordinate $r=R(t) \bar{r}$ in (52), we obtain

$$
\begin{aligned}
\mathrm{d} s^{2}= & c^{2}\left[1-\frac{\dot{R}^{2}(t) \bar{r}^{2}}{c^{2}}\right] \mathrm{d} t^{2}-2 R(t) \dot{R}(t) \bar{r} \mathrm{~d} \bar{r} \mathrm{~d} t \\
& -R^{2}(t)\left(\mathrm{d} \bar{r}^{2}+\bar{r}^{2} \mathrm{~d} \theta^{2}+\bar{r}^{2} \sin ^{2} \theta \mathrm{d} \varphi^{2}\right)
\end{aligned}
$$

It is completely different from the R-W metric (44) when $\kappa=0$. The metric (53) seems to be curved but is flat essentially. According to the principle of the Riemannian geometry, if we can find a method to turn a curved space into flat, the original space is flat essentially. If we can not find such method, the original space is a curved space in essence. It is obvious that we can not find a transformation to turn (52) into (45) when $\dot{R}(t) \neq 0$, the spatial part of (45) can not be flat!

On the other hand, the four dimensional metric in which three dimensional space has a constant curvature $\kappa$ is

$$
\begin{aligned}
\mathrm{d} S^{2} & =c^{2} \mathrm{~d} t^{2}-\mathrm{d} s^{2} \\
& =c^{2} \mathrm{~d} t^{2}-\left(\frac{\mathrm{d} r^{2}}{1-\kappa r^{2}}+r^{2} \mathrm{~d} \theta^{2}+r^{2} \sin ^{2} \theta \mathrm{d} \phi^{2}\right)
\end{aligned}
$$

by using co-moving coordinate in (54), we obtain

$$
\begin{aligned}
\mathrm{d} S^{2}= & {\left[c^{2}-\frac{\dot{R}^{2}(t)}{1-\kappa R^{2}(t) \bar{r}^{2}}\right] \mathrm{d} t^{2}-\frac{2 \dot{R}(t) R(t) \mathrm{d} t \mathrm{~d} \bar{r}}{1-\kappa R^{2}(t) \bar{r}^{2}} } \\
& -R^{2}(t)\left(\frac{\mathrm{d} \bar{r}^{2}}{1-\kappa R^{2}(t) \bar{r}^{2}}+\bar{r}^{2} \mathrm{~d} \theta^{2}+\bar{r}^{2} \sin ^{2} \theta \mathrm{d} \phi^{2}\right)
\end{aligned}
$$

Let $\kappa=0$, we reach (53) rather then (45). Therefore, if we use co-moving coordinate to describer the expansive universe in which the space is flat, we should use (53), rather than (45). If we describe the expansive universe with constant curvature, we should use (55), rather than (44).

Another result of using the R-W metric in cosmology is that it leads to the united universe time. In the R-W metric, $g_{00}=1$ indicates that we have the same time for any spatial point in the expansive universe. This obviously violates special relativity. Because there is a relative motion speed between two objects in the expansive universe, there exists time delay between them according to special relativity. It is actually the result of the Newtonian mechanics to use the united universe time in cosmology. This is another reason why we say that the Friedmann equation is equivalent to the Newtonian mechanics.

However, it is easy to prove that if we use flat spacetime metric (53) in the Einstein's equation of gravity, the Einstein's tensor would become zero with $R_{\mu v}=0$. In this way, we can not describe the gravity field of the expansive universe. Therefore, both the R-W metric and the flat space-time metric are unsuitable for cosmology. We should look for other proper methods to describe the expansive universe.

\subsection{Dynamic Energy Momentum Tensor Should Be Used in Cosmology}

The energy momentum tensor of ideal liquid is used in cosmology with the form

$$
T_{\mu v}=(\rho+p) U_{\mu} U_{v}-p g_{\mu v}
$$

Here $U_{\mu}(t)$ is the four dimensional velocity. In the standard cosmology, we take $U_{0}(t)=1$ and $U_{i}(t)=0$ with $T_{00}=\rho, T_{0 i}=0$. It means that we take static energy momentum tensor energy in the Einstein's equation of gravity without considering material's velocity.

This is an excessively simplified approximation. In fact, there exist relative velocities between materials and observers in the expansive universe. The most basic fact for cosmology is the Hubble's red shift, which is explained as the kinematical effect caused by relative velocities between observer and luminous material. If co-moving coordinate $r(t)=R(t) \bar{r}$, material's speed is $V(t)=\dot{R}(t) \bar{r}$ $\neq 0$. In fact, on the left side of the Fiedmann equation, we have $\dot{R}(t) \neq 0$. How can we take $\dot{R}(t) \neq 0$ on the 
right side of equation which contains energy momentum tensor? It is absolutely unjustifiable to use static energy momentum tensor to describe the expansive universe. In fact, if we use static energy momentum tensor to describe the expansive universe, what do we use to describer the static universe? This is a problem to make us embarrassing.

According general relativity, we can use arbitrary reference frame to describe the gravity field. By using common spherical coordinate system, the partial velocities of an object which moves along the radius direction are $V_{1}(t)$ $=\dot{r}(t), V_{2}(t)=V_{3}(t)=0$. The forth dimensional velocities are $(c=1)$.

$$
U_{0}=\frac{1}{\sqrt{1-\dot{r}^{2}}} \quad U_{1}=\frac{\dot{r}}{\sqrt{1-\dot{r}^{2}}} \quad U_{2}=U_{3}=0
$$

To simplify discussion below, we use the R-W metric and take $\kappa=0$. For the expansive universe with uniform distribution of material, dynamic energy momentum tensors are

$$
\begin{aligned}
& T_{00}=\frac{\rho+p}{1-\dot{r}^{2}}-p \\
& T_{01}=\frac{(\rho+p) \dot{r}}{1-\dot{r}^{2}} \\
& T_{11}=\frac{(\rho+p) \dot{r}^{2}}{1-\dot{r}^{2}}+p \\
& T_{22}=p r^{2} \\
& T_{33}=p r^{2} \sin ^{2} \theta \\
& T=g^{\mu v} T_{\mu v}=\rho-3 p
\end{aligned}
$$

Substituting them in the Einstein's equation of gravity

$$
R_{\mu v}+\lambda g_{\mu v}=-8 \pi G\left(T_{\mu v}-\frac{1}{2} T g_{\mu v}\right)
$$

We get the motion equations of cosmology

$$
\begin{gathered}
\frac{3 \ddot{r}}{r}+\lambda=-8 \pi G\left[\frac{\rho+p}{1-\dot{r}^{2}}+\frac{1}{2}(p-\rho)\right] \\
\lambda=8 \pi G \frac{(\rho+p) \dot{r}}{1-\dot{r}^{2}} \\
\frac{\ddot{r}}{r}+\frac{2 \dot{r}^{2}}{r^{2}}+\lambda=8 \pi G\left[\frac{(\rho+p) \dot{r}^{2}}{1-\dot{r}^{2}}+\frac{1}{2}(\rho-p)\right]
\end{gathered}
$$

Substitute $r(t)=R(t) \bar{r}$ in the formulas, we get

$$
\begin{gathered}
\frac{3 \ddot{R}}{R}+\lambda=-8 \pi G\left[\frac{\rho+p}{1-\dot{R}^{2} \bar{r}^{2}}+\frac{1}{2}(p-\rho)\right] \\
\lambda=8 \pi G \frac{(\rho+p) \dot{R} \bar{r}}{1-\dot{R}^{2} \bar{r}^{2}} \\
\frac{\ddot{R}}{R}+\frac{2 \dot{R}^{2}}{R^{2}}+\lambda=8 \pi G\left[\frac{(\rho+p) \dot{R}^{2} r^{2}}{1-\dot{R}^{2} \bar{r}^{2}}+\frac{1}{2}(\rho-p)\right]
\end{gathered}
$$

Take $\dot{R}=0$ on the right sides but not on the left sides, we obtain the Fiedmann equation. But we can not do it in this way. Because $\lambda$ is a constant, we have three ways to make (65) tenable. The first is to let $\dot{R}=\lambda=0$ which describes the static universe. By considering the observation fact of the Hubble redshift, this is improper. The second is to take simultaneously

$$
1-\dot{R}^{2} \bar{r}^{2}=A \text { and } 8 \pi G(\rho+p) \dot{R} \bar{r}=B
$$

Here $A$ and $B$ are constants. From (67), we obtain

$$
V=\dot{R} \bar{r}=\sqrt{1+A} \text { and } V=\dot{R} \bar{r}=\frac{B}{\rho+p}
$$

The result violates the Hubble law too. In addition, these velocities are inconsistent, so (68) is impossible. The third is to get the solution from (65)

$$
p=\frac{\left(1-\dot{R}^{2} \bar{r}^{2}\right) \lambda}{8 \pi G \dot{R} \bar{r}}-\rho
$$

Substitute (69) in (64) and (66), we have

$$
\begin{aligned}
& \frac{3 \ddot{R}}{R}+\lambda=-\frac{\lambda}{\dot{R} \bar{r}}\left(1+\frac{1-\dot{R}^{2} \bar{r}^{2}}{2}\right)+8 \pi G \rho \\
& \frac{\ddot{R}}{R}+\frac{2 \dot{R}^{2}}{R^{2}}+\lambda=\frac{\lambda}{\dot{R} \bar{r}}\left(\frac{1}{\dot{R} \bar{r}}-\frac{1-\dot{R}^{2} \bar{r}^{2}}{2}\right)+8 \pi G \rho
\end{aligned}
$$

by cancelling $\lambda$ from two formulas above, we obtain at last

$$
\begin{aligned}
& \left(3-\dot{R} \bar{r}-2 \dot{R}^{2} \bar{r}^{2}+2 \dot{R}^{3} \bar{r}^{3}\right) \frac{\ddot{R}}{R} \\
& +\left(1+2 \dot{R} \bar{r}+\dot{R}^{2} \bar{r}^{2}\right) \frac{\dot{R}^{3} \bar{r}}{R^{2}}-\left(1+\dot{R}^{3} \bar{r}^{3}\right) 8 \pi G \rho=0
\end{aligned}
$$

The equation becomes so complicated that it is impossible to solve actually. On the other hand, because the right hand sides of (64)-(66) contain $\bar{r}$, if $\rho(t)$ and $p(t)$ are still unrelated to $\bar{r}$, we should have $R=R(t, \bar{r})$ by solving the equations. The result contradicts with the original definition $r=R(t) \bar{r}$. In order to mate $R(t)$ unrelated to $\bar{r}, \rho$ and $p$ should be related to $\bar{r}$. In this way, the principle of cosmology can not hold again. The result means that we will be in dilemma if dynamic energy momentum tensor is used in cosmology.

Pioneers of cosmology must have considered this problem, so they had to use static energy momentum to establish the equation of cosmology. In the early stage of cosmology, the observed expansion speed of the universe was low, so the simplified motion equation could be suitable. When cosmology develops to now day's level, we observe cosmic phenomena which take place in the high speed expansive processes such as the high red shift of supernova. The simplified Friedmann equation becomes 
unsuitable so that many difficulties appear in the standard cosmology at present. This is the main reason why we have to introduce the hypothesis of the accelerating expansion of the universe, dark energy and non-baryon dark material.

\section{Velocity, Acceleration and Initial Conditions of the Universe Expansion}

\subsection{Velocity and Acceleration of the Universe Expansion}

Because (72) can not be solved practically when dynamic energy momentum tensor is considered, we have to look for other method to describe the expansive universe. We prove below that based on the revised formula (25), the high red shift of supernova can be explained well. Therefore, we do not need the hypothesis of dark energy and the universe accelerating expansion again.

In principle, we can take the CMB as static reference to describe the universe expansion. Practically, we take the earth as static reference frame for convenience. Suppose that the universe material is distributed with spherical symmetry and uniform density $\rho$. The static mass of sphere with radius $R$ is $M_{R}=4 \pi \rho R^{3} / 3$. Similarly we have $M_{r}=4 \pi \rho r^{3} / 3$ with radius $r$. According to the Newtonian theory, gravity acted on a small object located at point $r$ with mass $m_{0}$ is [6]

$$
F= \begin{cases}-\frac{G M_{R} m_{0}}{r^{2}} & r \geq R \\ -\frac{G M_{r} m_{0}}{r^{2}} & r \leq R\end{cases}
$$

The formulas indicate that when mass $m_{0}$ is located outsider the sphere with $r>R$, the gravity acted on it is equal to that when the spherical mass is centralized at the center of sphere. When mass $m_{0}$ is located inside the sphere with $r>R$, the gravity acted on it is only related to $M_{r}$, having nothing to do with the mass distributed outside the radius $r$.

Suppose that the universe expands along the direction of radius. In the process, angle momentum $L$ is equal to zero. We calculate gravity between a spherical shell with radius $R$ and an object located at point $r>R$ with static mass $m_{0}$ and speed $V_{r}$ along radius direction. Suppose that $V_{r}$ satisfies (22) approximately, we use (22) to describe object's effective mass. According to (23) and (25), we have

$$
\begin{aligned}
\mathrm{d} F & =-\frac{G m_{0} 4 \pi \rho R^{2}}{r^{2}} \sqrt{1-\frac{V_{R}^{2}}{c^{2}}} \sqrt{1-\frac{V_{r}^{2}}{c^{2}}} \mathrm{~d} R \\
& =-\frac{G m_{0} 4 \pi \rho}{r^{2}} \sqrt{1-\frac{V_{r}^{2}}{c^{2}}} \frac{R^{2} \mathrm{~d} R}{\sqrt{1+\alpha_{R} / R}}
\end{aligned}
$$

Here $\alpha_{R}=2 G M_{R} /\left(3 c^{2}\right)$. Let $\beta=8 \pi G \rho /\left(3 c^{2}\right)$, $\alpha_{R} / R=\beta R^{2}$, and taking the integral of (74), we get the total gravity that the expansive sphere with radius $R=r$ acts on an object located on the spherical surface with static mass $m_{0}$ and speed $V_{r}$

$$
\begin{aligned}
& F=-\frac{4 \pi G m_{0} \rho}{r^{2}} \sqrt{1-\frac{V_{r}^{2}}{c^{2}}} \int_{0}^{r} \frac{R^{2} \mathrm{~d} R}{\sqrt{1+\beta R^{2}}} \\
&=-\frac{2 \pi G m_{0} \rho}{\beta^{3 / 2} r^{2}} Q(\sqrt{\beta} r) \sqrt{1-\frac{V_{r}^{2}}{c^{2}}} \\
& Q(\sqrt{\beta} r)=\sqrt{\beta} r \sqrt{1+\beta r^{2}}-\ln \left(\sqrt{\beta} r+\sqrt{1+\beta r^{2}}\right)
\end{aligned}
$$

On the other hand, according to special relativity, we have

$$
F=\frac{\mathrm{d}}{\mathrm{d} t} \frac{m_{0} V_{r}}{\sqrt{1-V_{r}^{2} / c^{2}}}=\frac{m_{0} \ddot{r}}{\left(1-V_{r}^{2} / c^{2}\right)^{3 / 2}}
$$

Based on (76) and (77), we get the acceleration of an object located on spherical surface

$$
\ddot{r}=-\frac{2 \pi G \rho Q(\sqrt{\beta} r)}{\beta^{3 / 2} r^{2}}\left(1-\frac{V_{r}^{2}}{c^{2}}\right)^{2}
$$

The acceleration is just related to the mass inside the sphere, and unrelated to the mass outsider the sphere. We also consider (78) as the expansion speed of spherical surface with radius $r$. Let $V_{r} \rightarrow V$ and using relation $\ddot{r}=\mathrm{d} V / \mathrm{d} t=V \mathrm{~d} V / \mathrm{d} r$ in (78), we obtain

$$
\frac{1}{\left(1-V^{2} / c^{2}\right)^{2}} \frac{\mathrm{d} V^{2}}{\mathrm{~d} r}=-\frac{4 \pi G \rho}{\beta^{3 / 2} r^{2}} Q(\sqrt{\beta} r)
$$

Let $x=\sqrt{\beta} r$, we have

$$
x=\sqrt{\beta} r=\sqrt{\frac{8 \pi G \rho r^{2}}{3 c^{2}}}=\sqrt{\frac{2 G M}{c^{2} r}}=\sqrt{\frac{\alpha}{r}}
$$

In the expansion process of the universe, $\rho$ changes while spherical radius $r$ changes. But spherical mass $M$ is unchanged with $\alpha=$ constant. We have $\mathrm{d} x=\mathrm{d} \sqrt{\alpha / r}=$ $-\sqrt{\beta} \mathrm{d} r / 2$ or $\sqrt{\beta} \mathrm{d} r=-2 \mathrm{~d} x$. Suppose that initial radius is $r_{0}^{\prime}\left(x=x_{0}^{\prime}\right)$ and initial speed is $V_{0}^{\prime}$, substituting the relation into (79) and take the integral. Let

$$
Q_{1}(x)=3 \int \frac{Q(x)}{x^{2}} \mathrm{~d} x
$$

we get

$$
\frac{1}{1-V^{2} / c^{2}}=Q_{1}(r)-Q_{1}\left(r_{0}^{\prime}\right)+\frac{1}{1-V_{0}^{\prime 2} / c^{2}}
$$




$$
-Q_{1}\left(r_{0}^{\prime}\right)+\frac{1}{1-V_{0}^{\prime 2} / c^{2}}=C\left(r_{0}^{\prime}\right)+1
$$

we have

$$
\begin{aligned}
& \frac{1}{1-V^{2} / c^{2}}=Q_{1}(r)+C\left(r_{0}^{\prime}\right)+1 \\
& \frac{V}{C}=\sqrt{1-\frac{1}{Q_{1}(x)+C\left(r_{0}^{\prime}\right)+1}}
\end{aligned}
$$

Because (81) can not be integrated directly, we need approximate method. When $x$ is very small $(V / c \ll 1)$, by developing (76) into the Taylor's series in the region of $x=0$, we obtain

$$
\begin{aligned}
& Q(x)=\frac{2}{3} x^{3}-\frac{1}{5} x^{5}+\frac{3}{28} x^{7} \cdots \\
& Q_{1}(x)=x^{2}-\frac{3}{20} x^{4}+\frac{3}{56} x^{6} \cdots
\end{aligned}
$$

By considering (80), (87) becomes

$$
Q_{1}(x) \rightarrow Q_{1}(r)=\frac{2 G M_{0}}{c^{2} r}\left(1-\frac{3}{20} \frac{\alpha}{r}+\frac{3}{56}\left(\frac{\alpha}{r}\right)^{2}+\cdots\right)
$$

Substituting the formulas in (78) and (85), we obtain the formula of acceleration and speed of the universe expansion

$$
\begin{aligned}
\ddot{r}= & -\frac{G M_{0}}{r^{2}}\left(1-\frac{\alpha}{r}-\frac{1}{10}\left(\frac{\alpha}{r}\right)^{3 / 2}+\frac{3}{20}\left(\frac{\alpha}{r}\right)^{2}\right. \\
& \left.+\frac{29}{14}\left(\frac{\alpha}{r}\right)^{3 / 2}-\frac{3}{56}\left(\frac{\alpha}{r}\right)^{3} \cdots\right) \\
\frac{V}{c}= & \sqrt{Q_{1}(r)+C\left(r_{0}^{\prime}\right)} \\
= & \sqrt{\frac{2 G M_{0}}{c^{2} r}\left(1-\frac{3}{20} \frac{\alpha}{r}+\frac{3}{56}\left(\frac{\alpha}{r}\right)^{2}+\cdots\right)+C\left(r_{0}^{\prime}\right)}
\end{aligned}
$$

\subsection{Initial Condition of the Universe Expansion}

In the discussion above, we assume that material is only acted by gravity. However, practical situation is that strong, weak and electromagnetic interactions could not be neglected in the early phase of the universe during which material density was great. Even more, some unknown interaction may exist.

According to the theory of Einstein's theory, material may be compressed into infinite density by gravity. However, infinite density is unimaginable. In fact, the author had proved that due to use the improper boundary condition of flat space-time in the gravity theory of curved space-time, the current theory of singularity black hole is wrong. By strict calculation based on the Einstein's equation of gravity and curved boundary condition, singular black hole with infinity density do not exist [7,8]. By the same reasons, the fashionable idea that the universe originated from infinite small point is also impossible.

In order to avoid infinite density, we assume that there exist a certain mechanism so that material sphere with mass $M$ can only be compressed to a finite radius $r_{0}$. In this way, the motion equation of the universe expansion should be revised as

$$
m_{0} \ddot{r}=F(r)+F_{n}(r)
$$

Here $F(r)$ is gravity and $F_{n}(r)$ is the sum of other forces. For convenience, we simplify

$$
F_{n}(r)=\frac{m_{0}}{2} A(r) \sqrt{1-\frac{V^{2}}{c^{2}}} \delta\left(r-r_{0}^{\prime}\right)
$$

Here $A(r)$ is undetermined function. It corresponds to an infinite potential barrier with radius $r_{0}$ on which the spherical surface can not be contracted further. Meanwhile, by the action of $F_{n}(r)$ at the spherical surface with radius $r=r_{0}^{\prime}$, the process of contraction become expansion and the surface moves with a positive acceleration. When $r>r_{0}^{\prime}$, other forces become zero and only gravity acts. When $r_{0}^{\prime} \rightarrow 0$, it is just the so-called big bang of the universe from an infinite singular point. By considering (92), (79) becomes

$$
\frac{1}{\left(1-V^{2} / c^{2}\right)^{2}} \frac{\mathrm{d} V^{2}}{\mathrm{~d} r}=-\frac{4 \pi G \rho}{\beta^{3 / 2} r^{2}} Q(\sqrt{\beta} r)+A(r) \delta\left(r-r_{0}^{\prime}\right)
$$

The integral of (93) is

$$
\frac{1}{1-V^{2} / c^{2}}=Q_{1}(x)-Q_{1}\left(r_{0}^{\prime}\right)+A\left(r_{0}^{\prime}\right)+\frac{1}{1-V_{0}^{\prime 2} / c^{2}}
$$

Let

$$
-Q_{1}\left(r_{0}^{\prime}\right)+A\left(r_{0}^{\prime}\right)+\frac{1}{1-V_{0}^{\prime 2} / c^{2}}=K\left(r_{0}^{\prime}\right)+1
$$

$K\left(r_{0}^{\prime}\right)$ represents the initial condition of the universe expansion. For different objects located at different position $r$ now days, their initial positions $r_{0}^{\prime}$ and $K\left(r_{0}^{\prime}\right)$ are different. We will discuss how to decide $K\left(r_{0}^{\prime}\right)$ later. In this way, (94) becomes

$$
\frac{V}{c}=\sqrt{1-\frac{1}{Q_{2}(r)+K\left(r_{0}^{\prime}\right)+1}}
$$

Under the condition $V / c \ll 1$, by considering (86) and (88), the formulas of velocity and acceleration can be written as

$$
\begin{aligned}
\frac{V}{c} & =\sqrt{Q_{1}(r)+K\left(r_{0}^{\prime}\right)} \\
& =\sqrt{\frac{2 G M_{0}}{c^{2} r}\left(1-\frac{3}{20} \frac{\alpha}{r}+\frac{3}{56}\left(\frac{\alpha}{r}\right)^{2}+\cdots\right)+K\left(r_{0}^{\prime}\right)}
\end{aligned}
$$




$$
\begin{aligned}
\ddot{r}= & -\frac{G M_{0}}{r^{2}}\left(1-\frac{\alpha}{r}-\frac{1}{10}\left(\frac{\alpha}{r}\right)^{3 / 2}+\frac{3}{20}\left(\frac{\alpha}{r}\right)^{2}+\cdots\right) \\
& +K^{\prime}(r) \delta\left(r-r_{0}^{\prime}\right)
\end{aligned}
$$

Here $K^{\prime}(r)=K(r) \sqrt{1-V^{2} / c^{2}} / 2$. If expansive velocity is great, we should use (78) and (96) directly.

\section{Red Shifts of Cosmology and Hubble Diagram of Supernova}

\subsection{Red Shift of Cosmology}

According to the Doppler's formula, when celestial body moves along radius direction, we have relation between speed and red shift

$$
Z=\sqrt{\frac{1+V / c}{1-V / c}}-1
$$

Suppose that observer is located at the origin point of flat reference frame, the distance between observer and celestial body is $r(t)$ at moment $t$. In the expanding process of the universe, celestial body moves from $r_{1}$ to $r_{0}$ with $r_{0}>r_{1}$, while the light travels from $r_{1}$ to observer along opposite direction. Suppose light's speed is invariable in the process, we have following relation

$$
\Delta t=\int_{t_{1}}^{t_{0}} \mathrm{~d} t=\frac{r_{1}}{C}=\int_{r_{1}}^{r_{0}} \frac{\mathrm{d} r}{V}
$$

According to (96), we have

$$
r_{1}=\int_{r_{1}}^{r_{0}} \frac{\mathrm{d} r}{V / c}=\int_{r_{1}}^{r_{0}} \frac{\mathrm{d} r}{\sqrt{1-1 /\left(Q_{1}(r)+K\left(r_{0}^{\prime}\right)+1\right)}}
$$

The real distance between observer and celestial body is $r_{0}$ at present moment $t_{0}$. We know the universe material density $\rho_{0}$ at time $t_{0}$, but do not know its value $\rho_{0}$ at arbitrary time $t$. By considering relation $\rho_{0} r_{0}^{3}=$ $\rho r^{3}$, we write (80) as

$$
\sqrt{\beta} r=\sqrt{\frac{8 \pi G \rho r^{2}}{3 c^{2}}}=\sqrt{\frac{8 \pi G \rho_{0} r_{0}^{3}}{3 c^{2} r}}
$$

Using (102) in (101) and taking the integral, we can obtain the relation in principle

$$
r_{1}=f\left(r_{0}, K\left(r_{0}^{\prime}\right)\right)-f\left(r_{1}, K\left(r_{0}^{\prime}\right)\right)
$$

In the formulas above, $\rho_{0}, r_{1}$ and $Z$ are known through observations, but $\rho(t), r_{0}$ and $K\left(r_{0}^{\prime}\right)$ are unknown. By the relation $\rho(t) r^{3}=\rho_{0} r_{0}^{3}$, we can determinate $\rho(t)$. By connecting (100) and (103), we can determinate $r_{0}$ and $K\left(r_{0}^{\prime}\right)$. (101) can only be calculated by numerical method through computer. By taking $G=6.67 \times 10^{-11}, r_{0}=y_{0} \times$ $10^{26} \mathrm{~m}, r_{1}=y_{1} \times 10^{26} \mathrm{~m}$ and $\rho_{0}=b \times 10^{-26} \mathrm{~kg} / \mathrm{m}^{3}$, we have

$$
x=\sqrt{\frac{\alpha}{r}}=0.25 \sqrt{\frac{b y_{0}^{3}}{y}}
$$

We use $x$ as basic variable to calculate $y_{0}$ and $K\left(r_{0}^{\prime}\right)$ in which $b, Z$ and $y_{1}$ are input parameters. According to this paper, we actually deduce the initial situations of the universe expansion reversely based on the present observations of red shift and distances. In other words, as long as the initial conditions of the universe expansion are known, we can know its current situations.

\subsection{The Red Shift of Ia Supernova}

In Figure 1, the curved line with $\Omega_{0 m}=0.3$ and $\Omega_{\lambda}=$ 0.7 represents practical relation between red shift and distance of Ia supernova at the early period of time $t$. According to photometry measurement, the density of luminous material in the universe is about $\rho_{0} \approx 2 \times 10^{-28}$ $\mathrm{kg} / \mathrm{m}^{3}$ at present day. Because there exist a great mount of non-luminous material, we suppose that practical material is 10 times more than luminous material and let $\rho_{0} \approx 2 \times 10^{-27} \mathrm{~kg} / \mathrm{m}^{3}$. In Figure 1, we take $m_{B}=5.5+$ $5 \log \mathrm{d}_{L}$ in which $\mathrm{d}_{L}$ is luminosity distance with unit length $10^{6} p c=3.09 \times 10^{22} \mathrm{~m}$. But the concept of luminosity distance is unnecessary in this paper for our discussion is based on flat space-time. So we need to transform $\mathrm{d}_{L}$ to real distance $r$.

The curved line in Figure 2 shows the relations between red-shifts, distances and parameters of initial condition of Ia supernova. The vertical coordinate is the values of $K\left(r_{0}^{\prime}\right)$. The bottom horizontal coordinate is the value of red-shift. On the upside, under the line of horizontal coordinate are the values of distance $r$, above the line is the values of $r_{0}$. For $Z=1$ and $m_{B}=25$, we get $r_{1}=1.23 \times 10^{26} \mathrm{~m}$. By the numerical calculation, we obtain $r_{0}=1.91 \times 10^{26} \mathrm{~m}$ and $K\left(r_{0}^{\prime}\right)=-8.09 \times 10^{-2}$. For $Z$ $=0.5$ and $m_{B}=23.1$ corresponding to $r_{1}=0.67 \times 10^{26}$ $m$, we obtain $r_{0}=0.91 \times 10^{26}$ and $K\left(r_{0}^{\prime}\right)=3.41 \times 10^{-2}$. For $Z=0.1$ and $m_{B}=19.1$ corresponding to $r_{1}=0.15 \times 10^{26}$ $\mathrm{m}$, we obtain $r=0.16 \times 10^{26}$ and $K\left(r_{0}^{\prime}\right)=5.56 \times 10^{-3}$.

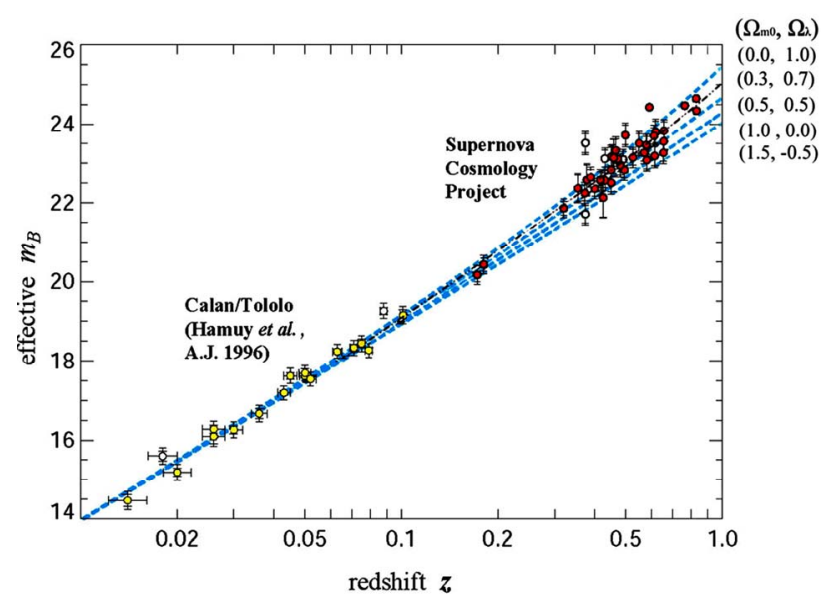

Figure 1. Hubble diagram for red shift and distance of Ia supernova (Cited from Ricss A.G. et al., 1998 ) 


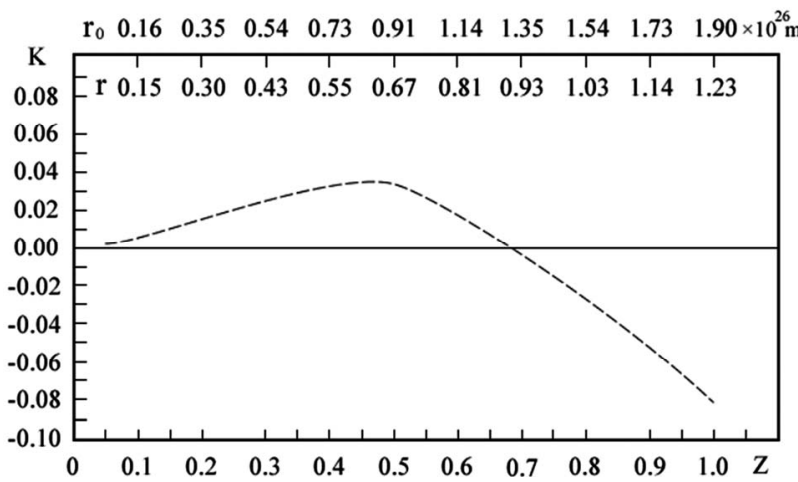

Figure 2. Relations between red-shifts, distances and initial parameters of Ia supernova.

In this way, we can explain the high red shift of Ia supernova well. The hypotheses of dark energy and the accelerating expansion of the universe become unnecessary. The universe began its expansion from a finite volume, rather than from a singularity.

\section{Revised Equation of Cosmology}

In order to compare with the equations of cosmology, we now transform (97) and (98) to the form of the Friedmann equation. Suppose that the universe is a uniform sphere with density $\rho=\rho(t)$. We define $r(t)=R(t, \bar{r}) \bar{r}$ in which $\bar{r}$ is a parameter unrelated with time. Let $t_{0}$ represent today's time, we have $r\left(t_{0}\right)=R\left(t_{0}, \bar{r}\right) \bar{r} \neq \bar{r}$ and can write (80) as

$$
\frac{\alpha}{r}=\frac{2 G M}{c^{2} r}=\frac{8 \pi G \rho}{3 c^{2}} r^{2}=b \rho R^{2} \bar{r}^{2}
$$

Here $b=8 \pi G /\left(3 c^{2}\right)$. Under the condition $V \ll c$, (100) can be written as

$$
\begin{aligned}
\frac{\ddot{R}}{R}= & -\frac{4 \pi G \rho}{3}\left(1-b \rho R \bar{r}-\frac{b^{3 / 2}}{10} \rho^{3 / 2} R^{2} \bar{r}^{2}\right. \\
& \left.+\frac{3 b^{2}}{20} \rho^{2} R^{3} \bar{r}^{3}+\cdots\right)+K^{\prime}(R \bar{r}) \delta\left(R-R_{0}^{\prime}\right)
\end{aligned}
$$

Similarly, let $k=-K\left(r_{0}^{\prime}\right)$, we can write (97) as

$$
\left(\frac{\dot{R}}{R}\right)^{2}+\frac{k}{R^{2}}=\frac{8 \pi G}{3} \rho\left(1-\frac{3 b}{20} \rho R \bar{r}+\frac{3 b^{2}}{56} \rho^{2} R^{3} \bar{r}^{3}+\cdots\right)
$$

On the other hand, the Friedmann equation containing cosmic constant $\lambda$ are

$$
\begin{aligned}
& \frac{\ddot{R}}{R}=-\frac{4 \pi G}{3}\left(\rho+3 p-2 \rho_{\lambda}\right) \\
& \left(\frac{\dot{R}}{R}\right)^{2}+\frac{k}{R^{2}}=\frac{8 \pi G}{3}\left(\rho+\rho_{\lambda}\right)
\end{aligned}
$$

Here $\rho_{\lambda}$ is considered as a constant energy density corresponding to vacuum and $\lambda$ in the current cosmol- ogy. Comparing (106) and (107) with (108) and (109), we have

$$
\begin{aligned}
& \rho_{\lambda}=-\frac{3 b}{20} \rho^{2} R \bar{r}+\frac{3 b^{2}}{56} \rho^{2} R^{3} \bar{r}^{3}+\cdots \\
& p=-\frac{23 b}{60} \rho^{2} R \bar{r}-\frac{b^{2}}{30} \rho^{5 / 2} R^{2} \bar{r}^{2}-\frac{b^{2}}{35} \rho^{2} R^{3} \bar{r}^{3}+\cdots
\end{aligned}
$$

It is obvious that after (110) and (111) are used, revised equations in this paper are with the same form with the Freidmann equation. The differences are that $\rho_{\lambda}$ is not a constant, $\rho_{\lambda}$ and $p$ are also related to $\bar{r}$. In order to be consistent with the observation of Ia supernova's red shift, the current theory have to assume $\rho+3 p-2 \rho_{\lambda}$ $<0$, so that we have to think that the universe is pushed by repulsive force and do accelerating expansion. According to this paper, we always have $\rho+3 p-2 \rho_{1}^{\prime}>0$, so there is no repulsive force and accelerating expansion again. Because the forms of differential equations (106) and (107) are very complex, it is more convenience for us to use (97) and (98) directly to do calculations.

\section{The Hubble Constant, Dark Material and the Universe Age}

\subsection{The Hubble Constant}

According to (97), we have

$$
Z=\frac{V}{c}=\sqrt{\frac{2 G M_{0}}{c^{2} r}\left(1-\frac{3}{20} \frac{\alpha}{r}+\frac{3}{56}\left(\frac{\alpha}{r}\right)^{2}+\cdots\right)+K\left(r_{0}^{\prime}\right)}
$$

Let $\rho_{e}$ represent the equivalent density of the universe

$$
\rho_{e}=\rho\left(1-\frac{3}{20} \frac{\alpha}{r}+\frac{3}{56}\left(\frac{\alpha}{r}\right)^{2}+\cdots\right)
$$

We get

$$
Z=\sqrt{\frac{8 \pi G \rho_{e} r^{2}}{3 c^{2}}+K\left(r_{0}^{\prime}\right)}=\frac{H r}{c}
$$

At present $t=t_{0}$ and $r=r_{0}$, the Hubble constant is

$$
H_{0}=\sqrt{\frac{8 \pi G \rho_{e 0}}{3 c^{2}}+\frac{K\left(r_{0}^{\prime}\right)}{r_{0}^{2}}}
$$

We see that $H_{0}$ is related to $K\left(r_{0}^{\prime}\right)$ and $r_{0}$, not a real constant even under condition $\alpha / r_{0} \ll 1$. This is the reason why we can not determinate the Hubble constant precisely up to present days.

In fact, only taking the first and last items in (98), we obtain the result of the Newtonian theory

$$
\frac{V}{c}=\sqrt{\frac{Z^{2}+2 Z}{Z^{2}+2 Z+2}}=\sqrt{\frac{8 \pi G \rho_{0} r^{2}}{3 c^{2}}+K\left(r_{0}^{\prime}\right)}
$$


Taking $\rho_{0}=2 \times 10^{-26} \mathrm{~kg} / \mathrm{m}^{3}$ and $\alpha / r=10^{-3}$, we get $r=2.84 \times 10^{23} \mathrm{~m}$. In cosmology, we generally take $H_{0}$ $=65 \mathrm{Km} \cdot \mathrm{s}^{-1} \cdot \mathrm{Mpc}^{-1}=2.0 \times 10^{-18} / \mathrm{s}$. We get $\mathrm{V} / \mathrm{c}=\mathrm{Z}=$ $1.89 \times 10^{-3}$ and $K\left(r_{0}^{\prime}\right)=2.57 \times 10^{-6}$ according to (116). In the calculation, we consider $r$ as the present position of luminous celestial, without considering its practical position to be $r_{0}$. For the situation with $Z=1$ and $r_{1}$ $=1.23 \times 10^{26} \mathrm{~m}$, according to (116), the result is $K\left(r_{0}^{\prime}\right)$ $=1.04 \times 10^{-17} \approx 0$. The result indicates that even though based on the Newtonian theory of gravity, we can also explain the high red shift of the Ia Supernova by taking different $K\left(r_{0}^{\prime}\right)$ for different objects. It is also unnecessary for us to introduce the concept of dark energy by introducing the effect of initial conditions.

For $Z=1.89 \times 10^{-3}$ and $r=2.84 \times 10^{23} \mathrm{~m}$, by using (96) for accurate numerical calculation, the result is $r_{0}=2.85$ $\times 10^{23}$ and $K\left(r_{0}^{\prime}\right)=3.0 \times 10^{-6}$ which is similar to that based on (116). But for the situations of high red shift, the differences of results are large.

\subsection{Non-Baryon Dark Material}

According to the theory of nuclei synthesis in cosmology, relative density of baryon is $\Omega_{b}=\rho_{b} / \rho_{h}$, in which $\rho_{b}$ is baryon's density and $\rho_{h}$ is total density of all material. We have relation [9]

$$
\Omega_{b 0} h^{2}=0.0037 \zeta \quad \zeta<9
$$

Take $H_{0}=65 \mathrm{Km} \cdot \mathrm{s}^{-1} \cdot \mathrm{Mpc}^{-1}$ which corresponds to $h=0.65$, we have $\rho_{h}=7.9 \times 10^{-27} \mathrm{~kg} / \mathrm{m}^{3}$ and $\Omega_{b}<0.08$. Practical observation is $\Omega_{m 0} \approx 0.30$, so the theory indicates that our universe is mainly composed of nonbaryon material. However, according to this paper, by considering the existence of parameter $K\left(r_{0}^{\prime}\right)$ in (115), it is enough for us to take $\rho_{h}=\rho_{0} \approx 2 \times 10^{-27} \mathrm{~kg} / \mathrm{m}^{3}$ and get $h=0.325$ and $\Omega_{b}<0.32$. We do not need the hypothesis of non-baryon dark material. At least, we do not need to assume that non-baryon dark material is $5 \sim 6$ times more than normal baryon material in the universe if non-baryon dark material exists actually.

\subsection{The Age of the Universe}

We consider the universe as a material sphere with radius $r_{0}^{\prime}=1.5 \times 10^{11} \mathrm{~m}$ at initial moment, which is about the distance between the sun and the earth. Long enough later, an observer located at the original point of reference frame receives the light omitted from a celestial body on the spherical surface with radius $r=1.23 \times 10^{26}$ $\mathrm{kg} / \mathrm{m}^{3}$ and find its red shift is $Z=1$ at time $t_{0}$. Suppose that the material density of the universe is $\rho_{0}=2 \times 10^{-27}$ at present, the initial density inside the sphere is $\rho_{0}^{\prime}=5.9 \times 10^{17} \mathrm{~kg} / \mathrm{m}^{3}$, equal to the density of neutron star. According to the calculation before, the celestial body has moved to the position $r_{0}=1.91 \times 10^{26}$ $\mathrm{m}$ at present moment. We consider this distance as the radius of the observable universe and substitute corresponding value $K\left(r_{0}^{\prime}\right)=-0.0809$ to following formula to calculate the time during which the universe expands from radius $r_{0}^{\prime}=1.5 \times 10^{11} \mathrm{~m}$ to $r_{0}=1.91 \times 10^{26} \mathrm{~m}$.

$$
\Delta t=\int_{t_{1}}^{t_{0}} \mathrm{~d} t=\int_{r_{0}^{\prime}}^{r_{0}} \frac{\mathrm{d} r}{V}=\int_{r_{0}^{\prime}}^{r_{0}} \frac{\mathrm{d} r}{c \sqrt{1-1 /\left(Q_{2}(r)-0.0809+1\right)}}
$$

The result is $\Delta t=30.8$ billion years. But this value is not sensitive to small initial radius. Taking $r_{1}=10^{20} \mathrm{~m}$, equal to the radius of the Milky Way galaxy, the result is the almost same. It means that the age of the universe mainly depends on the later expansive process.

Using (118) to calculates the time during which the universe radius expanses from $1.23 \times 10^{26} \mathrm{~m}$ to $1.95 \times$ $10^{26} \mathrm{~m}$, the result is 13 billion years, so the time during which the radius of the universe expanses from $1.5 \times 10^{11}$ $\mathrm{m}$ to $1.23 \times 10^{26} \mathrm{~m}$ is 17.8 billion years. This is just the universe age we consider at present. In the present cosmology, the universe age is estimated to be about $10 \sim 15$ billion years, too short to the formation of galaxies [10]. The problem does not exist according to this paper.

\section{Conclusions}

By transforming the geodesic equation of the Schwarzschild solution of the Einstein's equation into flat spacetime to describe, the revised Newtonian formula of gravity and the revised equation of cosmology are obtained. The singularity problem in the Einstein's theory of gravity described in curved space-time is eliminated thoroughly.

Because using two improper and approximate conditions, the Freidmann equation becomes the result of the Newtonian theory of gravity actually. It is only suitable to describe the low speed expansive processes of the universe, unsuitable to describe the high speed expansion. The equation of cosmology needs relativity revision.

By using the revised Newtonian formula of gravity, the revised equation of cosmology is obtained. The high red-shift of supernova can be well explained. It is unnecessary for us to introduce the hypotheses of the universe accelerating expansion and dark energy. It is also unnecessary for us to assume that non-baryon dark material is 5 6 times more than normal baryon dark material if it exists actually. Many problems existing in cosmology including the problem of the universe age can be resolved well.

In this way, the theory of gravity returns to the traditional form of dynamic description and becomes normal one. The revised equation can be used as the foundation of more rational cosmology.

\section{REFERENCES}

[1] N. Rosen, “General Relativity and Flat Space,” Physical 
Review, Vol. 57, No. 2, 1940, pp. 147-150. doi:10.1103/PhysRev.57.147

[2] Y. J. Wang and Z. M. Tang, "Theory and Effects of Gravitation,” Hunan Science and Technology Publishing Company, 1990, pp. 547-589.

[3] X. C. Mei, “The R-W Metric Has No Constant Curvature When Scalar Factor R(t) Changes with Time," International Journal of Astronomy and Astrophysics, Vol. 1, No. 4, 2011, pp. 177-182. doi:10.4236/ijaa.2011.14023

[4] E. A. Milne, “A Newtonian Expanding Universe,” General Relativity and Gravitation, Vol. 32, No. 9, 2000, pp. 1939-1948. doi:10.1023/A:1001997000979

[5] S. Weiberge, “Gravitation and Cosmology,” John Wiley and Sons, Inc., New York, 1984, p. 608.

[6] C. Kittel, W. D. Knight and M. A. Ruderman, "Mechan- ics,” Berkeley Physics Course, Vol. 1, McGraw-Hill, New York, 1973.

[7] X. C. Mei, "The Precise Inner Solutions of Gravity Field Equations of Hollow and Solid Spheres and the Theorem of Singularity,” International Journal of Astronomy and Astrophysics, Vol. 1, No. 3, 2011, pp. 109-116. doi:10.4236/ijaa.2011.13016

[8] X. C. Mei, "Singularities of the Gravitational Fields of Static Thin Loop and Double Spheres,” Journal of Cosmology, Vol. 13, Item 26, 2011.

[9] Y. Q. Yu, "Physical Cosmology,” Beijing University Publishing Company, Beijing, 2002, pp. 104, 184.

[10] M. Bolte and C. J. Hogan, "Conflict Over the Age of the Universe,” Nature, Vol. 376, 1995, pp. 399-402. doi:10.1038/376399a0 